\title{
MULTI-CLASS PROCEDURE FOR ANALYSIS OF ANTIBACTERIAL COMPOUNDS IN EGGS BY LIQUID CHROMATOGRAPHY-TANDEM MASS SPECTROMETRY
}

\author{
TOMASZ BŁĄDEK, ANDRZEJ POSYNIAK, ANNA GAJDA, \\ MAŁGORZATA GBYLIK, AND JAN ŻMUDZKI \\ Department of Pharmacology and Toxicology, \\ National Veterinary Research Institute, 24-100 Pulawy, Poland \\ tomasz.bladek@piwet.pulawy.pl
}

\begin{abstract}
A liquid chromatographic method coupled with tandem mass spectrometry for determination of residues of $\beta$-lactams, macrolides, tetracyclines, quinolones, sulfonamides, and lincosamides in eggs has been described. Analytes were isolated from egg samples by solvent extraction method and extracts were cleaned by filtration on OASIS HLB cartridges. The whole procedure was validated according to European Commission Decision 2002/657/EC. The recovery ranged between $86 \%$ and $110 \%$. The repeatability was below $16 \%$ and within-laboratory reproducibility was lower than $20 \%$. The method was successfully applied in the official control of antibacterial compounds residue in Poland.
\end{abstract}

Key words: eggs, antibiotics, HPLC-MS/MS.

Antibiotics and some other antimicrobial compounds are widely administered to food-producing animals for the purpose of prevention or treatment of bacterial diseases. Despite the positive effects of veterinary medicaments, inadequate use of antibiotics poses a potential health risk for consumers. For this reason the European Commission has set maximum residue limits (MRLs) for antibiotics in animal tissues, milk, and eggs (5). For some antibiotics such as chlortetracycline, oxytetracycline, tetracycline, and tylosin, MRL of $200 \mu \mathrm{g} / \mathrm{kg}$ in eggs have been set, whereas $150 \mu \mathrm{g} / \mathrm{kg}$ has been established for erythromycin, $50 \mu \mathrm{g} / \mathrm{kg}$ for lincomycin, and $500 \mu \mathrm{g} / \mathrm{kg}$ for neomycin. However, for other classes of antibacterials such as $\beta$-lactams, fluroquinolones, and sulfonamides, MRLs have not been set at all. Residues of antibiotics in eggs are controlled in National Residue Control Programme according to Council Directive 96/23 EC (6).

The screening for the presence of antibacterial compounds has been dominated by microbiological (3, 18), receptor-based (1), or immunological tests (12). They are easy to perform and inexpensive, but the main drawback of the common screening tests is their lack of specificity, thus positive results may not reveal, which antibiotic is present. Liquid chromatography coupled with UV and/or fluorometric (FL) detection has been applied for analysis of single antibiotic groups in eggs $(8,9,16,17)$. Over the past years, liquid chromatography - mass spectrometry (LC-MS) has been developed for the analysis of some groups of antibacterial compounds in eggs $(2,7,9-11,13,14,17)$. Liquid chromatography coupled with tandem mass spectrometry (LC-MS/MS) has become one of the most promising techniques for the analysis of antibiotics in eggs due to the determination of forbidden compounds that require limits detection as low as possible. Moreover, mass spectral analysis provides higher specificity than UV or FL detection. Furthermore, Commission Decision 2002/657/EC states that "methods based only on chromatographic analysis without the use of molecular spectrometric detection are not suitable for the use as confirmatory methods" (4).

The aim of this study was to develop a reliable and simple multi-class LC-MS/MS method for the analysis of antibiotics belonging to six different classes: $\beta$-lactams (penicillins and cephalosporins), macrolides, tetracyclines, quinolones, sulfonamides, and lincosamides. The procedure was validated according to European Commission Decision 2002/657/EC and was found suitable not only for identification of antibiotics but also for confirmation of positive results from immunochemical and microbiological screening systems used for routine control of antibiotics in eggs.

\section{Material and Methods}

Reagents. All organic solvents were HPLC grade and all chemicals were analytical grade. Acetonitrile, methanol, and sodium hydroxide were from J.T. Baker (the Netherlands). Heptafluorobutyric 
acid (HFBA), trifluoroacetic acid (TFA), and pentafluoropropionic acid (PFPA) were from SigmaAldrich (USA). Oxalic acid and disodium versenate dihydrate $\left(\mathrm{Na}_{2}\right.$ EDTA) were from POCH (Poland). Water was deionised $\left(>18 \mathrm{M} \Omega \mathrm{cm}^{-1}\right)$ in-house by Millipore system (Millipore, France). The SPE copolymer cartridges OASIS HLB (3 mL/60 mg) were obtained from Waters (USA) and PVDF syringe filters $(0.45 \mu \mathrm{m}$, $13 \mathrm{~mm}$ ) were received from Restek (USA).

Analytical standard and standard solutions. Amoxicillin (AMOX), ampicillin (AMPI), penicillin G (PEN G), penicillin V (PEN V), oxacillin (OXA), cloxacillin (CLOX), nafcillin (NAF), dicloxacillin (DICLOX), cephapirin (CFPI), ceftiofur (CFT), cefoperazone (CFPE), cephalexin (CFLE), cefquinome (CFQ), cefazolin (CFZ), cefalonium (CFLO), sulfaguanidine (SGU), sulfadiazine (SDZ), sulfathiazole (STZ), sulfamerazine (SME), sulfamethazine (SMT), sulfamethoxazole (SMA), sulfamethoxypyridazine (SMP), sulfamonomethoxine (SMM), sulfadoxine (SDX), sulfaquinoxaline (SQX), sulfadimethoxine (SDMX), tylosin (TYL), erythromycin (ERY), spiramycin (SPI), tilmicosin (TIL), josamycin (JOS), danofloxacin (DAN), difloxacin (DIF), enrofloxacin (ENR), ciprofloxacin (CIP), flumequine (FLU), sarafloxacin (SAR), marbofloxacin (MAR), norfloxacin (NOR), oxolinic acid (OXO), nalidixic acid (NAL), chlortetracycline (CTC), tetracycline (TC), doxycycline (DC), oxytetracycline (OTC), and sulfaphenazole (internal standard) were from Sigma-Aldrich (USA).

Stock standard solutions $\left(1 \mathrm{mg} \mathrm{mL}^{-1}\right)$ were prepared by weighing $10.0 \pm 0.1 \mathrm{mg}$ of each standard substances, followed by quantitative transfer to a $10 \mathrm{ml}$ volumetric flask and filling to volume with methanol or water. The standards of macrolides, tetracyclines, quinolones, sulfonamides, and lincomycin were dissolved in methanol, whereas $\beta$-lactams were dissolved in ultra pure water and stored at $-20^{\circ} \mathrm{C}$. Working standard solutions and mixed standard solutions were prepared by diluting suitable aliquot of stock standard in ultra pure water and stored at approximately $4^{\circ} \mathrm{C}$.

Sample preparation and extraction. Samples were prepared from fresh eggs. Egg shell was discarded and combined yolk and albumen were homogenised at room temperature and frozen at $-20^{\circ} \mathrm{C}$ until the time of analysis.

An aliquot (1 g) of previously homogenised whole egg was placed in a $50 \mathrm{ml}$ polypropylene centrifuge tube and $50 \mu \mathrm{l}$ of $2 \mu \mathrm{g} \mathrm{mL}^{-1}$ solution of sulfaphenazole (internal standard) was added. At that time, the control samples were spiked with mixed standard solution. One $\mathrm{ml}$ of $0.02 \mathrm{M}$ of oxalic acid $(\mathrm{pH}$ 4), $0.5 \mathrm{ml}$ of $0.1 \mathrm{M} \mathrm{Na}_{2}$ EDTA and $8 \mathrm{ml}$ of acetonitrile were added to the centrifuge tubes and the samples were homogenised with Vortex mixer. The mixtures were centrifuged for $10 \mathrm{~min}$ at 4,200 x $g$ and supernatants were cleaned by passing through an OASIS HLB cartridges. The cleaned extracts were evaporated to dryness in nitrogen evaporator at $45^{\circ} \mathrm{C}$. The dry residues were reconstituted in $1 \mathrm{ml}$ of $0.025 \%$ HFBA in water and filtered through $0.45 \mu \mathrm{m}$ PVDF filters.
LC-MS/MS. An Agilent Series 1200 HPLC system (Agilent Technologies, Germany) was connected to a AB Sciex API 4000 quadrupole mass spectrometer (AB Sciex, Canada). The mass spectrometer was operated in electrospray positive ionisation mode (ESI+) and two multiple reaction monitoring (MRM) transitions were monitored in order to give four identification points in compliance with Commission Decision 2002/657/EC. The mass spectrometer settings were optimised both with direct infusion of working standard solution from a syringe pomp and with LC injection. The following mass spectrometer parameters were used: resolution Q1 and Q3 = unit; nebuliser gas = 40 psi; auxiliary gas $=50 \mathrm{psi}$, curtain gas $=20 \mathrm{psi}$; collision gas $=3 \mathrm{psi}$; ion spray voltage $=5,500$; temperature $=500{ }^{\circ} \mathrm{C}$. The fragmentation reactions used for monitoring were selected on the basis of their significance in products ion spectra. The Analyst 1.5.1 software controlled the LCMS/MS system and processed the data.

The chromatographic separation was performed on a Luna octadecyl (C18) column (150 x $2.0 \mathrm{~mm}, 3$ $\mu \mathrm{m})$ (Phenomenex ${ }^{\circledR}$, USA) coupled with octadecyl guard column $(2 \times 4 \mathrm{~mm})\left(\right.$ Phenomenex ${ }^{\circledR}$, USA). The mobile phase consisted of solvent A: $0.025 \%$ of HFBA in water $(\mathrm{v} / \mathrm{v})$ and solvent B: acetonitrile. The elution was performed in a gradient mode; the mobile phase starting conditions were $95 \%$ of eluent A for 2 min and then decreased to $10 \%$ within $9 \mathrm{~min}$. This composition was stable for $3 \mathrm{~min}$ and then increased to $95 \%$ of eluent A. With the following equilibration time of $10 \mathrm{~min}$, the resulting total run time was $24 \mathrm{~min}$. The column was operated at $35^{\circ} \mathrm{C}$ with a flow rate of $0.25 \mathrm{~mL} \mathrm{~min}^{-1}$, and the injection volume was $30 \mu 1$.

Evaluation of the procedure. The whole procedure was validated according to recommendations of the European Commission Decision 2002/657/EC. The validation study was performed in terms of selectivity, specificity, accuracy, precision (repeatability and within-laboratory reproducibility), as well as calculation of decision limit $(\mathrm{CC} \alpha)$ and detection capability (CC $\beta)$. Five-points matrix-matched calibration curves spiked at the levels $0,0.5,1.0,1.5$, and 2.0 times of the validation level (VL) were obtained by plotting the response of respective analyte/internal standard peak area ratio versus the analyte/internal standard concentration. Validation level defined in this paper as the MRL for drugs with MRL or specific level of interest for drugs without MRL equalled $40 \mu \mathrm{g} / \mathrm{kg}$.

To evaluate possible interferences encountered in the method, the specificity of method was verified by analysing 20 different blank samples. Precision (repeatability and within-laboratory reproducibility) was determined by the repeated analysis $(n=6)$ of egg samples spiked with analytes at concentrations corresponding to $0.5,1.0$, and 1.5 times of the validation level, from run-to run during $1 \mathrm{~d}$ and $3 \mathrm{~d}$, respectively. Precision was evaluated by calculating the relative standard deviation (RSD) of the results obtained for each level of the target compound. The recoveries were evaluated in the same experiment as repeatability by comparing the measured concentrations to the fortified 
concentrations of the samples. The $\mathrm{CC} \alpha$ and $\operatorname{CC} \beta$ were determined by the matrix calibration curve procedure.

\section{Results}

The developed procedure was designed to obtain qualitative and quantitative surveillance information about the antibacterial compounds belonging to different chemical groups and to analyse simultaneously the same analytical protocol. Identification was carried out by retention times, identification points of each analyte as required by the EU validation criteria, and relative ion ratio of selected MRM transitions. The optimal conditions obtained for analysis of antibacterial compounds by mass spectrometry including precursor ion (Q1), product ion (Q3), dwell time, declustering potential (DP), entrance potential (EP), collision energy (CE), cell exit potential (CXP), and retention time (RT) are listed in Table 1.

Gradient elution utilising an octadecyl chromatographic column was used for separation of multi-class drugs. At the first step of the study, trifluoroacetic acid (TFA), pentafluoropropionic acid (PFPA) and heptafluorobutyric acid (HFBA) solutions were studied to evaluate the most appropriate one. The chromatograms obtained showed that HFBA provided more symmetric peaks than the other ion-pairing reagents and the best peak shapes were obtained at $0.025 \%$ HFBA. The optimal separation of $\beta$-lactams, sulfonamides, macrolides, tetracyclines, fluoroquinolones, and lincomycin was obtained with the column Luna C18 (150 x $2.0 \mathrm{~mm}, 3 \mu \mathrm{m})$ coupled with mobile phase consisted of acetonitrile and $0.025 \%$ HFBA in water.

The validation parameters were estimated on the basis of in-house validation concept in accordance with Commission Decision 2002/657/EC. Matrix calibration curves were used for quantification in order to reach a high accuracy. The basic value for the calculation of the parameters is the level of interest, i.e. the VL. The specificity of the method was checked by analysing blank egg samples of different origins and the specificity was $100 \%$ for all analytes, as no peak was detected in these samples at the retention time corresponding to each analyte. The recoveries were in the range between $86 \%$ and $110 \%$. Precision (repeatability and withinlaboratory reproducibility) of the procedure, as well as decision limit $(\mathrm{CC} \alpha)$ and detection capability $(\mathrm{CC} \beta)$ estimated for spiked egg samples are listed in Table 2. Fig. 1 demonstrates typical chromatograms from the analysis of antibacterial compounds belonging to $\beta$ lactams, sulfonamides, macrolides, tetracyclines, fluoroquinolones, and lincomycin (MRM 1 is presented). The stability of individual stock standard solutions of analytes stored at $-20^{\circ} \mathrm{C}$ was maintained for at least 6 months, with the exception of penicillins, which were stable for 3 months. Working standard solutions and mixed standard solutions stored approximately at $4^{\circ} \mathrm{C}$ were stable for at least 1 month. The stability of the analytes in eggs matrix stored at $20^{\circ} \mathrm{C}$ was at least 4 weeks.

\section{Discussion}

The proper analysis of the residues of the veterinary drugs in eggs is very difficult, because of a very complicated composition of matrices of eggs. The albumen (egg white) is a polar medium with glycoproteins, while the ovum (egg yolk) is non polar because the presence of lipoproteins. Other analytical difficulties are associated with the different physicochemical properties of the antibacterial compounds included in this study.

The significant binding between the lipoprotein and drugs contributes to poor isolation of analytes from matrices of eggs. To obtain optimal conditions for isolation of multi-class antibacterial compounds from eggs, the sample must be buffered before extraction procedure. Additionally, it must be mentioned that some classes of antibacterial compounds (tetracyclines, fluoroquinolones) can form complex with metal ions. Therefore, suitable reagents have to be included to the analytical procedure.

The extraction procedure, described in "Material and Methods", was adopted after extensive investigation of methods for the extraction of analytes of the interest from eggs. It was based on the researches by Garrido Frenich et al. (7) for the extraction of several classes of veterinary drugs in eggs. In our preliminary comparative studies, it was found that the use of acetonitrile extraction after treatment of sample with oxalic buffer and $\mathrm{Na}_{2}$ EDTA solution was sufficient for simultaneous isolation of penicillins, cephalosporins, macrolides, fluoroquinolones, sulfonamides, tetracyclines, and lincomycin from eggs' matrix. Only aminoglycosides, because of their polarity, were not isolated from eggs' matrix with satisfactory results, so it was decided not to include this group of antibacterial compound to the developed procedure and prepare the separate analytical protocol.

Raw extracts obtained from eggs' matrix were very dirty, so the clean-up step with solid-phase extraction cartridge was included to the analytical protocol. The sample clean-up is not complicated and makes the method fast and simple. This enabled the analysis of a large number of samples in a one working day. Sensitivity achieved by this simple process was sufficient to determine the analytes at the concentration levels of interest. Five points matrix-matched standards calibration curve ensured correct quantification of samples, if possible ion suppression effects were corrected.

Based on previous results obtained in our laboratory (15), the Luna C18 column was chosen in the present study. One liquid chromatography gradient schedule was developed to separate of $\beta$-lactams, macrolides, fluoroquinolones, sulfonamides, tetracyclines, and lincomycin. Chromatographic separation is particularly important when isobaric compounds such as sulfamethoxypyridazine and sulfamonomethoxine, sulfadoxine and sulfadimethoxine, flumequine and oxolinic acid, tetracycline and doxycycline are present. In our method, these analytes were successfully separated, as shown in Fig. 1. 


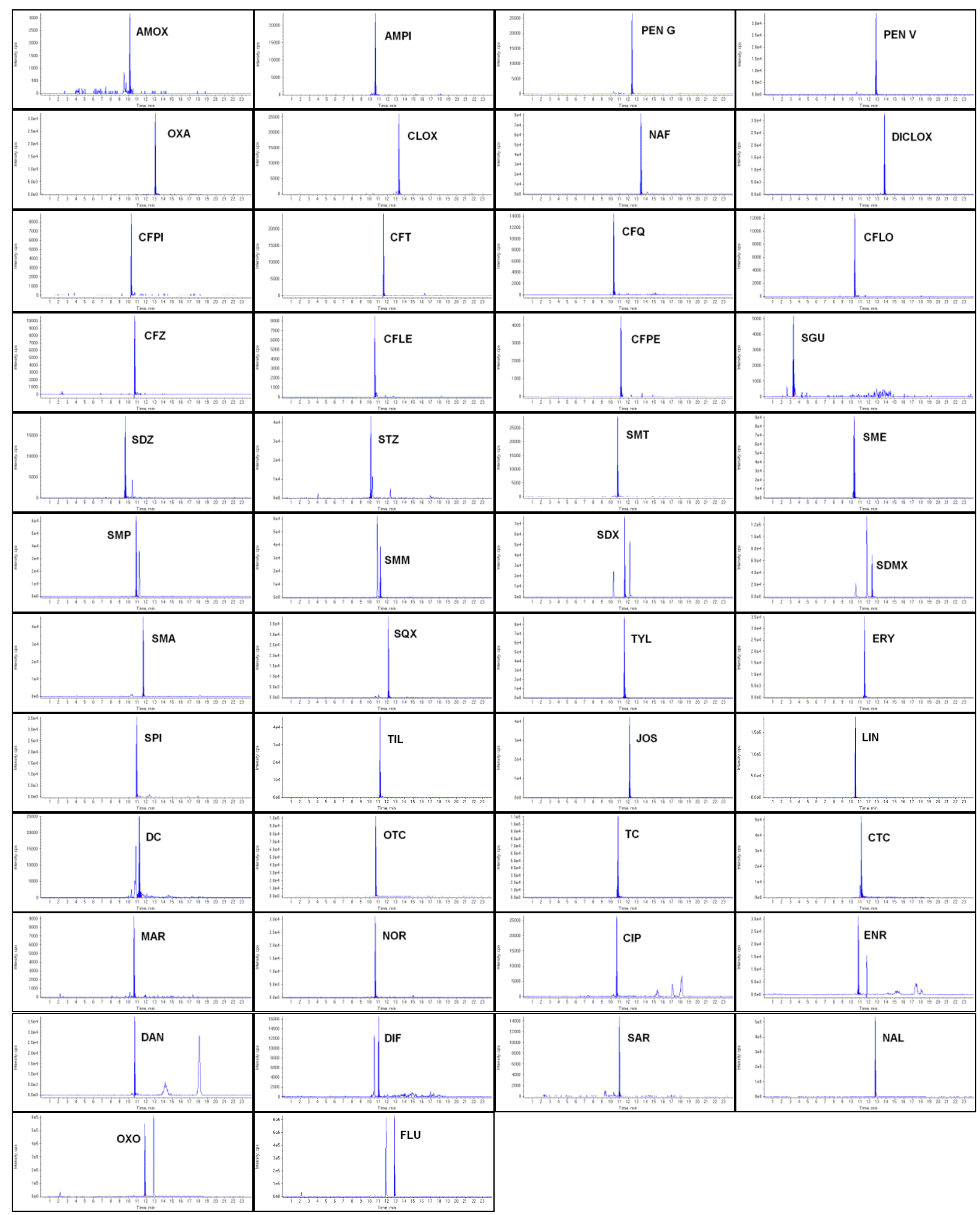

Fig. 1. Chromatograms for MRM 1 of egg sample spiked with each of the 46 target drugs at 0.5 VL concentration. 
Table 1

Mass spectrometry parameters for quantitative and qualitative analysis of antibacterial compounds

\begin{tabular}{|c|c|c|c|c|c|c|c|c|}
\hline Analyte & $\begin{array}{c}\mathrm{RT} \\
(\mathrm{min})\end{array}$ & $\begin{array}{c}\text { Precursor } \\
(\mathrm{m} / \mathrm{z})\end{array}$ & $\begin{array}{c}\text { Products } 1 / 2 \\
(\mathrm{~m} / \mathrm{z})\end{array}$ & $\begin{array}{l}\text { DP } \\
(\mathrm{V})\end{array}$ & $\begin{array}{l}\text { EP } \\
(V)\end{array}$ & $\begin{array}{c}\text { CE } 1 / 2 \\
(\mathrm{~V})\end{array}$ & $\begin{array}{c}\text { CXP 1/2 } \\
(\mathrm{V})\end{array}$ & $\begin{array}{l}\begin{array}{c}\text { Dwell } \\
(\mathrm{ms})\end{array}\end{array}$ \\
\hline Amoxicillin & 10.2 & 366 & $208 / 349$ & 45 & 10 & $18 / 14$ & $4 / 8$ & 5 \\
\hline Ampicillin & 10.6 & 350 & $106 / 160$ & 58 & 5 & $27 / 19$ & $5 / 15$ & 5 \\
\hline Penicillin G & 12.4 & 335 & $160 / 176$ & 60 & 5 & $17 / 19$ & $15 / 17$ & 5 \\
\hline Penicillin V & 12.8 & 351 & $160 / 114$ & 54 & 5 & $17 / 48$ & $15 / 10$ & 5 \\
\hline Oxacillin & 13.1 & 402 & $160 / 243$ & 50 & 10 & $18 / 18$ & $15 / 15$ & 5 \\
\hline Cloxacillin & 13.4 & 436 & $160 / 277$ & 50 & 10 & $20 / 20$ & $15 / 15$ & 5 \\
\hline Nafcillin & 13.5 & 415 & 199/171 & 50 & 10 & $20 / 50$ & $15 / 15$ & 5 \\
\hline Dicloxacillin & 13.8 & 470 & $160 / 311$ & 50 & 10 & $20 / 20$ & $15 / 15$ & 5 \\
\hline Cephapirin & 10.3 & 424 & $152 / 124$ & 50 & 10 & $35 / 70$ & $15 / 15$ & 5 \\
\hline Cefoperazone & 11.2 & 646 & $530 / 143$ & 60 & 10 & $17 / 50$ & $15 / 15$ & 5 \\
\hline Cephalexin & 10.6 & 348 & $158 / 106$ & 50 & 10 & $10 / 23$ & $15 / 15$ & 5 \\
\hline Cefquinome & 10.3 & 529 & $134 / 125$ & 50 & 10 & $25 / 75$ & $15 / 15$ & 5 \\
\hline Cefazolin & 10.7 & 455 & $323 / 156$ & 50 & 10 & $15 / 23$ & $15 / 15$ & 5 \\
\hline Cefalonium & 10.4 & 459 & $337 / 152$ & 46 & 5 & $16 / 28$ & $8 / 15$ & 5 \\
\hline Ceftiofur & 11.6 & 524 & $241 / 125$ & 50 & 10 & $25 / 70$ & $15 / 15$ & 5 \\
\hline Sulfaguanidine & 3.1 & 215 & $156 / 108$ & 20 & 10 & $20 / 30$ & $15 / 15$ & 5 \\
\hline Sulfadiazine & 9.6 & 251 & $156 / 108$ & 53 & 10 & $22 / 30$ & $15 / 15$ & 5 \\
\hline Sulfathiazole & 10.1 & 256 & $156 / 108$ & 53 & 10 & $20 / 34$ & $15 / 15$ & 5 \\
\hline Sulfamerazine & 10.3 & 265 & $156 / 108$ & 45 & 10 & $25 / 37$ & $15 / 10$ & 5 \\
\hline Sulfamethazine & 10.8 & 279 & $156 / 108$ & 50 & 10 & $25 / 36$ & $15 / 15$ & 5 \\
\hline Sulfamethoxazole & 11.7 & 254 & $156 / 108$ & 50 & 10 & $23 / 35$ & $15 / 10$ & 5 \\
\hline Sulfamethoxypyridazine & 10.9 & 281 & $156 / 108$ & 60 & 10 & $25 / 35$ & $15 / 15$ & 5 \\
\hline Sulfamonomethoxine & 11.2 & 281 & $156 / 108$ & 50 & 10 & $23 / 37$ & $15 / 15$ & 5 \\
\hline Sulfadoxine & 11.6 & 311 & $156 / 108$ & 60 & 10 & $25 / 40$ & $15 / 15$ & 5 \\
\hline Sulfadimethoxine & 12.2 & 311 & $156 / 108$ & 50 & 10 & $23 / 37$ & $15 / 15$ & 5 \\
\hline Sulfaquinoxaline & 12.1 & 301 & $156 / 108$ & 50 & 10 & $23 / 40$ & $15 / 15$ & 5 \\
\hline Tylosin & 11.6 & 916 & $174 / 772$ & 110 & 10 & $52 / 42$ & $10 / 20$ & 5 \\
\hline Erythromycin & 11.5 & 734 & $158 / 576$ & 75 & 10 & $42 / 27$ & $15 / 15$ & 5 \\
\hline Spiramycin & 10.9 & 843 & $174 / 540$ & 120 & 10 & $52 / 44$ & $16 / 14$ & 5 \\
\hline Tilmicosin & 11.2 & 869 & $174 / 696$ & 135 & 10 & $61 / 56$ & $17 / 18$ & 5 \\
\hline Josamycin & 12.2 & 828 & $174 / 229$ & 80 & 10 & $46 / 44$ & $16 / 14$ & 5 \\
\hline Danofloxacin & 10.7 & 358 & $340 / 255$ & 60 & 10 & $33 / 50$ & $15 / 15$ & 5 \\
\hline Difloxacin & 11.0 & 400 & $382 / 356$ & 50 & 10 & $30 / 28$ & $9 / 8$ & 5 \\
\hline Enrofloxacin & 10.8 & 360 & $342 / 286$ & 72 & 10 & $30 / 50$ & $15 / 15$ & 5 \\
\hline Ciprofloxacin & 10.7 & 332 & $314 / 231$ & 61 & 10 & $30 / 47$ & $15 / 15$ & 5 \\
\hline Flumequine & 12.9 & 262 & $244 / 202$ & 44 & 10 & $25 / 45$ & $15 / 15$ & 5 \\
\hline Sarafloxacin & 11.0 & 386 & $368 / 348$ & 50 & 10 & $31 / 46$ & $8 / 8$ & 5 \\
\hline Marbofloxacin & 10.6 & 363 & $345 / 320$ & 70 & 10 & $30 / 22$ & $15 / 15$ & 5 \\
\hline Norfloxacin & 10.6 & 320 & $302 / 231$ & 60 & 10 & $33 / 50$ & $15 / 15$ & 5 \\
\hline Oxolinic acid & 11.9 & 262 & $244 / 216$ & 53 & 10 & $25 / 40$ & $15 / 15$ & 5 \\
\hline Nalidixic acid & 12.7 & 233 & $215 / 187$ & 42 & 10 & $30 / 35$ & $15 / 15$ & 5 \\
\hline Chlortetracycline & 11.1 & 479 & $444 / 462$ & 56 & 10 & $31 / 25$ & $14 / 16$ & 5 \\
\hline Tetracycline & 10.8 & 445 & $410 / 427$ & 36 & 10 & $27 / 19$ & $12 / 18$ & 5 \\
\hline Doxycycline & 11.2 & 445 & $428 / 154$ & 55 & 10 & $25 / 42$ & $15 / 15$ & 5 \\
\hline Oxytetracycline & 10.7 & 461 & $426 / 443$ & 41 & 10 & $27 / 19$ & $14 / 20$ & 5 \\
\hline Lincomycin & 10.4 & 407 & $126 / 359$ & 74 & 10 & $36 / 28$ & $7 / 8$ & 5 \\
\hline Sulfaphenazole (IS) & 12.2 & 315 & 156 & 50 & 10 & 30 & 15 & 5 \\
\hline
\end{tabular}

$\mathrm{RT}$ - retention time, DP - declustering potential, FP - focusing potential, CE - collision energy, CXP - cell exit potential. 
Table 2

Validation parameters for antibiotics in spiked egg samples at $1 \mathrm{VL}$

\begin{tabular}{|c|c|c|c|c|c|c|}
\hline Analyte & $\begin{array}{c}\text { MRL/VL } \\
(\mu \mathrm{g} / \mathrm{kg})\end{array}$ & $\begin{array}{c}\text { Repeatability } \\
(\%)\end{array}$ & $\begin{array}{c}\text { Within-laboratory } \\
\text { reproducibility } \\
(\%)\end{array}$ & $\begin{array}{c}\mathrm{CC} \alpha \\
(\mu \mathrm{g} / \mathrm{kg})\end{array}$ & $\begin{array}{c}C C \beta \\
(\mu \mathrm{g} / \mathrm{kg})\end{array}$ & $\begin{array}{c}\text { Recovery } \\
(\%)\end{array}$ \\
\hline Amoxicillin & 40 & 15.9 & 18.5 & 30 & 43 & 97 \\
\hline Ampicillin & 40 & 9.9 & 14.2 & 30 & 42 & 103 \\
\hline Penicillin $\mathrm{G}$ & 40 & 7.7 & 11.9 & 28 & 34 & 94 \\
\hline Penicillin V & 40 & 8.2 & 15.5 & 30 & 41 & 87 \\
\hline Oxacillin & 40 & 12.7 & 14.8 & 30 & 39 & 90 \\
\hline Cloxacillin & 40 & 15.3 & 17.3 & 30 & 41 & 88 \\
\hline Nafcillin & 40 & 13.8 & 18.3 & 29 & 38 & 86 \\
\hline Dicloxacillin & 40 & 12.6 & 13.0 & 28 & 37 & 101 \\
\hline Cephapirin & 40 & 13.9 & 19.4 & 29 & 42 & 102 \\
\hline Cefoperazone & 40 & 11.9 & 14.6 & 30 & 41 & 102 \\
\hline Cephalexin & 40 & 7.5 & 10.3 & 27 & 34 & 108 \\
\hline Cefquinome & 40 & 11.2 & 14.8 & 27 & 36 & 105 \\
\hline Cefazolin & 40 & 14.6 & 16.5 & 28 & 38 & 103 \\
\hline Cefalonium & 40 & 10.9 & 17.2 & 27 & 38 & 102 \\
\hline Ceftiofur & 40 & 7.8 & 11.1 & 28 & 36 & 106 \\
\hline Sulfaguanidine & 40 & 10.0 & 10.3 & 27 & 35 & 102 \\
\hline Sulfadiazine & 40 & 12.4 & 15.9 & 29 & 39 & 102 \\
\hline Sulfathiazole & 40 & 10.8 & 12.5 & 25 & 33 & 105 \\
\hline Sulfamerazine & 40 & 13.6 & 15.1 & 28 & 39 & 105 \\
\hline Sulfamethazine & 40 & 11.2 & 12.4 & 27 & 35 & 102 \\
\hline Sulfamethoxazole & 40 & 10.6 & 12.3 & 25 & 33 & 103 \\
\hline Sulfamethoxypyridazine & 40 & 9.8 & 11.4 & 29 & 39 & 105 \\
\hline Sulfamonomethoxine & 40 & 9.7 & 13.7 & 28 & 37 & 103 \\
\hline Sulfadoxine & 40 & 8.5 & 13.8 & 26 & 34 & 110 \\
\hline Sulfaquinoxaline & 40 & 9.3 & 10.0 & 26 & 32 & 100 \\
\hline Sulfadimethoxine & 40 & 11.0 & 12.3 & 26 & 33 & 101 \\
\hline Tylosin & 200 & 9.2 & 11.5 & 231 & 275 & 100 \\
\hline Erythromycin & 150 & 10.3 & 11.8 & 178 & 223 & 107 \\
\hline Spiramycin & 40 & 11.0 & 11.7 & 26 & 31 & 99 \\
\hline Tilmicosin & 40 & 13.3 & 14.8 & 25 & 33 & 103 \\
\hline Josamycin & 40 & 9.1 & 158 & 28 & 36 & 98 \\
\hline Danofloxacin & 40 & 11.6 & 14.1 & 27 & 36 & 103 \\
\hline Difloxacin & 40 & 12.3 & 16.9 & 28 & 38 & 103 \\
\hline Enrofloxacin & 40 & 11.3 & 14.5 & 27 & 36 & 103 \\
\hline Ciprofloxacin & 40 & 13.3 & 13.5 & 28 & 37 & 104 \\
\hline Flumequine & 40 & 9.3 & 14.5 & 26 & 34 & 98 \\
\hline Sarafloxacin & 40 & 14.4 & 14.6 & 27 & 35 & 103 \\
\hline Marbofloxacin & 40 & 12.7 & 16.8 & 27 & 36 & 103 \\
\hline Norfloxacin & 40 & 14.0 & 15.2 & 27 & 37 & 104 \\
\hline Oxolinic acid & 40 & 10.1 & 12.9 & 26 & 34 & 107 \\
\hline Nalidixic acid & 40 & 7.0 & 12.0 & 25 & 31 & 101 \\
\hline Chlortetracycline & 200 & 9.8 & 12.8 & 232 & 285 & 102 \\
\hline Tetracycline & 200 & 8.1 & 12.8 & 235 & 281 & 101 \\
\hline Doxycycline & 40 & 11.6 & 17.6 & 28 & 39 & 103 \\
\hline Oxytetracycline & 200 & 9.5 & 9.6 & 238 & 277 & 103 \\
\hline Lincomycin & 50 & 11.3 & 15.4 & 58 & 75 & 105 \\
\hline
\end{tabular}


The procedure was successfully validated according to Commission Decision 2002/657/EC. The method is implemented in the National Residue Control Programme in Poland for confirmation of positive results from immunochemical and microbiological screening tests and for routine control of antibiotics in eggs (about 50 samples per year is analysed by this procedure). Among the samples analysed in 2011, three samples contained doxycycline or enrofloxacin. This was confirmed using the criteria described in "Material and Methods". Since doxycycline and enrofloxacin are forbidden to use in laying hens, the obtained results indicate that these samples might have been derived from farms using inappropriate drugs.

\section{References}

1. Alfredsson G., Branzell C., Granelli K., Lundstrom A.: Simple and rapid screening and confirmation of tetracyclines in honey and egg by a dipstick test and LCMS/MS. Anal Chim Acta 2005, 529, 47-51.

2. Bogialli S., D’Ascenzo G., Di Corcia A., Laganà A., Tramontana G.: Simple assay for monitoring seven quinolone antibacterials in eggs: extraction with hot water and liquid chromatography coupled to tandem mass spectrometry. Laboratory validation in line with the European Union Commission Decision 657/2002/EC. J Chomatogr A 2009, 1216, 794-800.

3. Cantwell H., O'Keefee M.: Evaluation of the Premi Test and comparison with the One-Plate Test for the detection of antimicrobials in kidney. Food Add Contam 2006, 23, 120-125.

4. Commission Decision 2002/657/EC of August 2002 implementing Council Directive 96/23/EC concerning the performance of analytical methods and the interpretation of results. Off J Eur Commun 2002, L 221, 8-36.

5. Commission Regulation (UE) $37 / 2010$ of $22^{\text {nd }}$ December 2009 on pharmacologically active substances and their classification regarding maximum residue limits in foodstuffs of animal origin. Off J Eur Commun 2010, L 15, 1-72.

6. Council Directive 96/23/EC of April 1996 on measures to monitor certain substances and residues thereof in live animals and animal products and repealing Directives 85/358/EEC and 86/469/EEC and Decisions 89/18/EEC and 91/664/EEC. Off J Eur Commun 1996, L 125, 10-32.

7. Garrido Frenich A., del Mar Aguilera-Luiz M., Martinez Vidal J.L., Romero-Gonzalez R.: Comparison of several extraction techniques for multiclass analysis of veterinary drugs in eggs using ultra-high pressure liquid chromatography-tandem mass spectrometry. Anal Chim Acta 2010, 661, 150-160.

8. Hassouan M.K., Ballesteros O., Taoufiki J., Vìlchez J.L., Cabrera-Aguilera M., Navalón A.: Multiresidue determination of quinolone antibacterials in eggs of laying hens by liquid chromatography with fluorescence detection. J Chromatogr B 2007, 852, 625-630.

9. Heller D.N., Ngoh M.A., Donoghue D., Podhorniak L., Righter H., Thomas M.H.: Identification of incurred sulfonamide residues in eggs: method confirmation by liquid chromatography-tandem mass spectrometry and quantitation by liquid chromatography with ultraviolet detection. J Chomatogr B 2002, 774, 39-52.

10. Heller D.N., Nochetto C.B., Rummel N.G., Thomas M.H.: Development of multiclass method for drug residue in eggs: hydrophilic solid-phase extraction cleanup and liquid chromatography/tandem mass spectrometry analysis of tetracycline, fluoroquinolone, sulfonamide, and $\beta$-lactam residues. J Agric Food Chem 2006, 54, 5267-5278.

11. Jia X., Shao B., Wu Y., Yang Y., Zhang J.: Simultaneous determination of tetracylines and quinolones antibiotics in eggs by ultra-performance liquid chromatographyelectospray tandem mass spectrometry. J AOAC Int 2008, 91, 461-468.

12. McGrath T., Baxter A., Ferguson J., Haughey S., Bjurling P.: Multi sulfonamide screening in porcine muscle using a surface plasmon resonance biosensor. Anal Chim Acta 2005, 529, 123-127.

13. Mol H.G.J., Plaza-Bolaños P., Zomer P., de Rijk T.C., Stolker A.A.M., Mulder P.P.J.: Toward a generic extraction method for simultaneous determination of pesticides, mycotoxins, plant toxins, and veterinary drugs in feed and food matrixes. Anal Chem 2008, 80, 94509460.

14. Peters R.J.B., Bolck Y.J.C., Rutgers P., Stolker A.A.M., Nielen M.W.F.: Multi-residue screening of veterinary drugs in egg, fish and meat using high-resolution liquid chromatography accurate mass time-of-flight mass spectrometry. J Chromatogr A 2009, 1216, 8206-8216.

15. Posyniak A., Zmudzki J., Mitrowska K.: Dispersive solid-phase extraction for the determination of sulfonamides in chicken muscle by liquid chromatography. J Chromatogr A 2005, 1087, 259-264.

16. Schneider M.J., Donoghue D.J.: Multiresidue determination of fluoroquinolones in eggs. J AOAC Int 2000, 83, 1306-1312.

17. Schneider M.J., Donoghue D.J.: Multiresidue determination of fluoroquinolone antibiotics in eggs using liquid chromatography-fluorescence-mass spectroscopy. Anal Chim Acta 2003, 483, 39-49.

18. Stead S., Sharman M., Tarbin J.A., Gibson E., Richmond S., Stark J., Geijp E.: Meeting maximum residue limits: an improved screening technique for the rapid detection of antimicrobial residues in animal food products. Food Add Contam 2004, 21, 216-221. 